\title{
GENERACIÓN DE ESTADOS EXTENDIDOS EN MODELOS DESORDENADOS CON IMPUREZAS TIPO DELTA
}

\author{
María Onell S. $\quad$ Edmundo Lazo N. ${ }^{1}$ \\ Recibido el 03 de enero de 2002, aceptado el 19 de agosto de 2002
}

\begin{abstract}
RESUMEN
En este trabajo se estudia un sistema unidimensional con impurezas tipo delta con amplitudes al azar. Dicho sistema se diluye introduciendo, alternadamente, impurezas tipo delta con amplitud constante. Mediante la técnica de decimación se demuestra la existencia de dos conjuntos infinitos de estados deslocalizados. La comprensión de los mecanismos que permiten la transición metal-aislador, abre la posibilidad de llegar a fabricar dispositivos electrónicos donde dicha transición es relevante.
\end{abstract}

Palabras claves: Desorden, deslocalización, Anderson

\begin{abstract}
In this work we study a 1D system with delta type impurities with random amplitudes. This system is diluted introducing, in alternating way, delta type impurities with constant amplitudes. Using the decimation technique, we demonstrate the existence of two infinite sets of delocalized states. The understanding of the mechanism, which allows the metalinsulator transition, opens the possibility of building electronic devices where that transition is relevant.
\end{abstract}

Keywords: Disorder, deslocalization, Anderson

\section{INTRODUCCIÓN}

El descubrimiento que los sistemas unidimensionales desordenados pueden tener transiciones metal-aislador constituye un avance teórico crucial que tiene gran relevancia desde el punto de vista tecnológico, debido a que abre las posibilidades de fabricación de dispositivos con bordes de movilidad predeterminados, los cuales pueden ser usados como ventanas filtradores en estructuras electrónicas, acústicas y fotónicas.

En términos teóricos, las propiedades de localización de modelos tight-binding fueron estudiadas inicialmente por Anderson [1], quien mostró que ciertos estados resultan ser localizados debido al desorden. La posterior generalización de este trabajo permitió probar que en una dimensión, todos los estados son localizados para cualquier grado de desorden.

El estudio del grado de localización de la función de onda cuántica en sistemas desordenados con correlación en el desorden ha sido objeto de gran atención en el último tiempo [2-20]. Además de incorporar la correlación en el desorden, últimamente se ha introducido el proceso de "dilución" de los sistemas desordenados [21], [22]. En general, la dilución consiste en introducir elementos no desordenados entre cada par de sitios desordenados.

Recientemente hemos realizado un estudio sistemático del efecto que produce el proceso de dilución en sistemas unidimensionales tight-binding desordenados tipo Anderson [23-27]. En términos generales, se obtiene un conjunto finito de estados extendidos cuyo número y posición energética dependen de la energía por sitio de los átomos que diluyen al sistema desordenado y del número de dichos átomos que se introducen entre dos sitios vecinos desordenados.

El estudio de sistemas discretos tiene la ventaja de facilitar el estudio numérico, sin embargo, es necesario hacerse una pregunta fundamental: ¿dependen los resultados obtenidos de la naturaleza discreta del hamiltoniano? Este aspecto fue discutido por Bellisard [28] y Kohmoto [29], quienes obtuvieron una ecuación

\footnotetext{
1 Universidad de Tarapacá, Facultad de Ciencias, Departamento de Física, Arica, Chile, elazo@uta.cl, monell@uta.cl.
} 


$$
H \phi(x)=E \phi(x)
$$

se escribe:

$$
z_{n} \phi_{n}-\phi_{n-1}-\phi_{n+1}=0
$$

donde

$$
z_{n}=\left(2 \cos q+\frac{\lambda_{h}}{q} \sin q\right)
$$

acá $\lambda_{n}$ rotula las amplitudes de las deltas, $\phi_{n}=\phi\left(x_{n}\right), \quad q=d \sqrt{E}, \quad$ donde $\quad d=\left(x_{n+1}-x_{n}\right)$ representa la distancia entre dos sitios vecinos. La ecuación (2) es exactamente equivalente a la ecuación

En este trabajo consideramos que la distancia entre dos sitios vecinos es constante, $d=1, \mathrm{y}$ que en las posiciones pares de la red unidimensional, las amplitudes $\lambda_{2 n}$ del potencial tipo delta se obtienen al azar del conjunto $\left[-\frac{w}{2}, \frac{w}{2}\right]$, donde $w$ es pequeño. En las posiciones impares las amplitudes del potencial tipo delta tienen un valor constante $\lambda_{2 n \pm 1}=\lambda_{0}=c t e$. De este modo se consigue diluir el sistema desordenado al azar. Sin embargo, el sistema completo sigue siendo desordenado, y por lo tanto, en principio, todos sus autoestados deberían ser localizados, a excepción de los estados extendidos que fueron encontrados por primera vez por Ishii [32], asociados a la existencia de la correlación espacial de la distribución de impurezas en la red, como puede verse al hacer $\sin q=0$ en el mapa de Poincaré (2). Dicha condición significa que existen algunas energías $E_{n}=n^{2} \pi^{2}, \quad n \geq 1$, para las cuales el desorden se elimina de la ecuación (2), quedándonos con un sistema puro, y en consecuencia todos los estados que cumplen con dicha condición son estados extendidos o deslocalizados. Estos son los únicos estados extendidos conocidos en este modelo, hasta la fecha [32], [33].

Escribamos $z_{n}$ dado por (3) en la forma sencilla siguiente:

$$
z_{n}=a+b \lambda_{n}
$$

con

$$
a=2 \cos q ; \quad b=\frac{\sin q}{q} .
$$

Aplicando el mapa de Poincaré (2) a los sitios pares e impares de la red unidimensional, podemos escribir:

$$
\begin{aligned}
& z_{0} \phi_{2 n-1}-\phi_{2 n-2}-\phi_{2 n}=0, \\
& z_{2 n} \phi_{2 n}-\phi_{2 n-1}-\phi_{2 n+1}=0 \\
& z_{0} \phi_{2 n+1}-\phi_{2 n}-\phi_{2 n+2}=0 .
\end{aligned}
$$

En la primera y la tercera ecuaciones hemos escrito $z_{2 n \pm 1}=z_{0}$ porque la amplitud de los potenciales tipo delta en los sitios impares es constante.

Realizando un proceso de decimación, las ecuaciones (6), (7) y (8) pueden ser escritas en la forma:

$$
\left[\begin{array}{ll}
z_{0} & z_{2 n}-2
\end{array}\right] \phi_{2 n}-\phi_{2 n-2}-\phi_{2 n+2}=0
$$

Estas ecuaciones representan completamente al sistema desordenado diluido original. Sin embargo, se pueden visualizar dos formas distintas de eliminar el desorden de modo de lograr, para algunas energías especiales, un sistema ordenado y por lo tanto que sus autoestados presenten el carácter de extendidos:

- $\quad$ Caso en que $b=0$.

En este caso la ecuación (9) queda:

$$
\left(a^{2}-2\right) \phi_{2 n}-\phi_{2 n-2}-\phi_{2 n+2}=0,
$$

y la solución resulta ser el mismo conjunto infinito numerable de energías $E_{n}=n^{2} \pi^{2}, \quad n \geq 1$ obtenido 
por Ishii [32] para el caso del sistema sin dilución. Nuevamente vemos que para cada una de estas energías $E_{n}$ la función de onda es extendida ya que el desorden ha desaparecido.

- $\quad$ Caso en que $z_{0}=\left(a+b \lambda_{0}\right)=0$.

En este caso la ecuación (9) queda:

$$
2 \phi_{2 n}+\phi_{2 n-2}+\phi_{2 n+2}=0
$$

Aquí vemos que existe un conjunto infinito de valores $q_{n}$ para los cuales se cumple la condición $z_{0}=0$, que corresponde a la ecuación no lineal:

$$
q+\left(\frac{\lambda_{0}}{2}\right) \operatorname{tg}(q)=0
$$

a partir de las cuales podemos encontrar las energías $E_{n}$ de los nuevos es tados extendidos: $E_{n}=q_{n}^{2}$.

En el presente modelo continuo desordenado y diluido, hemos encontrado las condiciones para las cuales el desorden puede ser eliminado sistemáticamente. Sin embargo, existe una condición adicional que debe cumplirse en un sistema unidimensional para que las soluciones pertenezcan al espectro de autovalores del sistema (ver ecuación (9)):

$$
\left|z_{0} z_{2 n}-2\right| \leq 2
$$

Así, cuando $b=0$, la condición (11) se escribe $\left|2 \cos ^{2} q-1\right| \leq 1, \quad \mathrm{y}$ todos los $q_{n}=n \pi$, son soluciones físicas. Por otra parte, cuando $z_{0}=\left(a+b \lambda_{0}\right)=0$ se tiene $|-2| \leq 2$, cumpliéndose trivialmente la condición (11). De este modo, todos los $q_{n}=\sqrt{E_{n}}$ que son solución de la ecuación (10), corresponden a autoestados extendidos del sistema unidimensional continuo desordenado y diluido.

En resumen, hemos demostrado analíticamente, que el mecanismo de dilución de un sistema continuo desordenado permite generar un nuevo conjunto infinito de energías, cada una de las cuales corresponde a nuevos estados deslocalizados o extendidos. En este punto es importante destacar que dichos estados extendidos no tienen una representación en términos de funciones de Bloch, ya que se trata de estados extendidos correspondientes a un sistema altamente desordenado. La generalización del presente modelo para el caso de dilución con mayor número de átomos es directa, y se espera que aparezca un número finito de ecuaciones no lineales similar a la ecuación (10), cada una de las cuales genera un conjunto infinito energías correspondientes a estados extendidos o deslocalizados.

Los estados extendidos del sistema tienen directa relación con las propiedades de conducción de los sistemas desordenados. En consecuencia, el estudio de sistemas desordenados y la comprensión de los mecanismos que permiten generar transiciones desde estados localizados a estados extendidos y viceversa, resulta de gran importancia desde el punto de vista tecnológico, ya que abre las posibilidades de construcción de dispositivos electrónicos de diversas aplicaciones.

\section{AGRADECIMIENTOS}

Se agradece el financiamiento parcial de este trabajo a través del proyecto 4743/01 de la Dirección de n y Postgrado de la UTA.

\section{REFERENCIAS}

[1] P. W. Anderson; Phys. Rev. Vol.109, pp 1492, 1958.

[2] J. C. Flores; J. Phys. Condens. Matter Vol. 1, pp. 8471, 1989.

[3] D. H. Dunlap, Phillips P. Kundu; Phys. Rev. B Vol. 40,pp 10999, 1989.

[4] D. H. Dunlap, H. L. Wu, Phillips P.; Phys. Rev. Lett. Vol. 65,pp. 88, 1990.

[5] H. L. Wu, Phillips P.; Phys. Rev. Lett. Vol. 66, pp. 1366, 1991.

[6] H. L. Wu, W. Goff, Phillips P.; Phys. Rev. B Vol. 45, pp. 1623, 1992.

[7] Phillips P., H. L. Wu; Science, Vol 252, pp. 1805, 1991.

[8] S. Gangopadhyay, A. K. Sen, J. Phys; Condens. Matter Vol. 4, pp. 9939, 1992.

[9] P. K. Datta, D. Giri, K. Kundu; Phys. Rev. B Vol. 47, pp. 10727, 1993.

[10] A. Sánchez, F. Domínguez-Adame, Phys. J.; A: Math. Gen. Vol. 27, pp. 3725, 1994. 
[11] A. Sánchez, E. Maciá, F. Domínguez-Adame; Phys. Rev. B Vol. 49,pp. 147, 1994.

[12] E. Diez, A. Sánchez, F. Domínguez-Adame; Phys. Rev. B Vol. 50,pp. 14359, 1994.

[13] F. Domínguez-Adame, E. Diez, A. Phys. Rev. B Vol. 51, pp. 8115, 1995.

[14] M. Hilke; Phys J. A, Math. Gen. Vol. 27, pp. 4773, 1994.

[15] M. Hilke, J. C. Flores; Phys. Rev. B Vol. 55, pp. $10625,1997$.

[16] E. Lazo, M. E. Onell; Revista Mexicana de Física, Vol. 44, Suplemento 1, pp. 52, 1998.

[17] F. A. B. F. De Moura., M. L. Lyra; Phys. Rev. Lett. Vol. 81, pp. 3735, 1998.

[18] F. M. Izrailev, A. A. Krokhin; Phys. Rev. Lett. Vol. 82, pp 4062, 1999.

[19] V. Bellani, E. Diez, R. Hey, L. Toni, L. Tarricone, G. B. Parravicini, F. Domínguez-Adame, R. Gómez-Alcalá; Phys. Rev. Lett. Vol. 82, pp. 4062, 1999.

[20] U. Kuhl, F. M. Izrailev, A. A. Krokhin, H. J. Stöckmann; Appl. Phys. Lett. Vol. 77, pp. 633, 2000.

[21] M. Hilke, J. Phys. A; Math. Gen. Vol. 30, L367. 1997.

[22] W. Deng; Physica B, Vol. 279, pp 224, 2000.

[23] E. Lazo, M. E. Onell; Physica B Vol. 299, pp. 173, 2001.

[24] E. Lazo, M. E. Onell; Physics Letters A Vol. 283, pp. 376, 2001.

[25] M. E. Onell, E. Lazo; "Extended states in chains of polyhedra with Anderson disorder", Revista Mexicana de Física, en imprenta, 2002.

[26] E. Lazo, M. E. Onell; "Delocalized states in random size diluted Anderson clusters", en arbitraje en Physical Review B, 2002.

[27] E. Lazo, M. E. Onell; “Appearance of delocalized states in diluted quasi-one-dimensional Anderson Systems" en arbitraje en Journal physics A: Math. Gen., 2002.

[28] J. Bellisard, A. Bovier and J. M. Ghez; Rev. Math. Phys. 4, Vol.1, 1992.

[29] M. Kohmoto; Phys. Rev. B Vol. 34, pp. 5043, 1986.

[30] A. Sánchez, F. Domínguez-Adame; Phys J. A; Math. Gen. Vol. 27, pp 3725, 1994.
[31] A. Sánchez, E. Maciá, F Domínguez-Adame; Phys. Rev. B Vol. 49, pp. 147, 1994.

[32] K. Ishii; Suppl. Prog. Theo. Phys. Vol. 53, pp. 77, 1973.

[33] A. Sánchez F. Domínguez-Adame; J. Phys. Rev. B Vol. 49,pp. 147, 1994. 